\title{
Essai de Lutte Contre les Principaux ravageurs de la courgette par l'utilisation d'extraits de Ocimum canum Sims, à Korhogo dans le Nord de la Côte d'Ivoire
}

\author{
Klana Koné, Doctorant \\ Université Félix Houphouët-Boigny, \\ Laboratoire de Zoologie et Biologie Animale \\ Yalamoussa Tuo, Maître-Assistant \\ Université Peleforo Gon Coulibaly, Département de Biologie Animale \\ Mouhamed Cissé, Maître-Assistant \\ Université Peleforo Gon Coulibaly, Département de Biochimie et Génétique \\ Hervé Kouakou Koua, Professeur Titulaire \\ Université Félix Houphouët-Boigny, \\ Laboratoire de Zoologie et Biologie Animale
}

Doi:10.19044/esj.2019.v15n24p72～URL:http://dx.doi.org/10.19044/esj.2019.v15n24p72

\section{Résumé}

Les producteurs de courgette du département de Korhogo utilisent les pesticides de synthèse de façon déraisonnée et incontrôlée pour protéger leurs parcelles contre l'action des insectes ravageurs. Dans le but de trouver des méthodes de lutte alternative aux produits de synthèse, l'efficacité des extraits aqueux, hydro-alcoolique et l'huile essentielle de Ocimum canum a été testée sur les principaux insectes ravageurs de la courgette (les pucerons, les aleurodes, les chrysomèles et les mouches). Les essais ont été menés sur une parcelle expérimentale de $81 \mathrm{~m} 2$ logée au sein du jardin botanique de l'Université Peleforo Gon Coulibaly de Korhogo. Ces substances biologiques ont permis de contrôler efficacement les principaux ravageurs, et de réduire leurs dégâts. L'extrait hydro-alcoolique $(67 \mathrm{~g} / \mathrm{L})$ et l'extrait aqueux $(67 \mathrm{~g} / \mathrm{L})$, ont été les plus efficaces sur les pucerons et les aleurodes. Au niveau des chrysomèles, l'extrait hydro-alcoolique $(67 \mathrm{~g} / \mathrm{L})$ et l'huile essentielle (333 $\mathrm{g} / \mathrm{L}$ ) étaient les plus efficaces. Cependant tous les extraits de plante et l'insecticide de synthèse (Cypercal $50 \mathrm{~g} / \mathrm{L}$ ) n'ont pas eu d'impact dans la réduction des dégâts des mouches de la courgette.

Mots clés : Courgette, Insectes ravageurs, Extraits de plante, Ocimum canum 


\title{
Trial to Struggle Against the Main Zucchini Pests by Using Extracts from Ocimum Canum Sims, in Korhogo in Northern Côte d'Ivoire
}

\author{
Klana Koné, Doctorant \\ Université Félix Houphouët-Boigny, \\ Laboratoire de Zoologie et Biologie Animale \\ Yalamoussa Tuo, Maître-Assistant \\ Université Peleforo Gon Coulibaly, Département de Biologie Animale \\ Mouhamed Cissé, Maître-Assistant \\ Université Peleforo Gon Coulibaly, Département de Biochimie et Génétique \\ Hervé Kouakou Koua, Professeur Titulaire \\ Université Félix Houphouët-Boigny, \\ Laboratoire de Zoologie et Biologie Animale
}

\begin{abstract}
Zucchini producers in the Korhogo Department use synthetic pesticides unreasonably and uncontrollably to protect their plots against the action of insect pests. In order to find alternative control methods for synthetic pesticides, the effectiveness of aqueous extracts, hydro-alcoholic and essential oils of Ocimum canum has been tested on the main insect pests of zucchini (aphids, whiteflies, beetles and flies). Tests were conducted on an experimental plot of $81 \mathrm{~m}^{2}$ housed in the botanical garden of the Peleforo Gon Coulibaly University of Korhogo. These biological substances have effectively controlled the main pests, and reduced their damage. The hydroalcoholic extract and the aqueous extract were the most effective on aphids and whiteflies. At the level of the beetles, the hydroalcoholic extract and the essential oils were the most effective. However, all plant extracts and synthetic pesticide hadn't an impact in reducing the damage of zucchini flies.
\end{abstract}

Keywords: Zucchini, Insect pests, Plant extracts, Ocimum canum

\section{Introduction}

Les cultures maraichères sont pratiquées aujourd'hui dans toutes les zones de production agricole en Côte d'Ivoire sur des petites surfaces (KoffieBipko et Yéo, 2016). En plus des légumes habituellement cultivés (Aubergine, Tomate, Choux, Salade), la culture de la courgette, Cucurbita pepo est en plein essor dans le département de Korhogo. Selon les estimations de la FAO en 
2014, la production de courgettes en Côte d'Ivoire était de 19296 Tonnes. La courgette se produit en moyenne en 45 jours. Ce court cycle de production fait de ce légume un excellent palliatif de légumes tels que l'aubergine (4 mois), ou le chou (3mois) dans la préparation des sauces.

Cependant, l'augmentation de la production est limitée par plusieurs contraintes, notamment l'action des insectes nuisibles (Rajendran et Singh, 2016). Dans le département de Korhogo, les principaux ravageurs de la courgette sont les pucerons, les aleurodes, les chrysomèles et les mouches de la courgette (Koné et al., 2018 ; Koné et al., 2019a). Ces ravageurs causent des dégâts sur tous les organes de la plante (Racines, Tiges, Feuilles, Fleurs et fruits) et certains de ces ravageurs transmettent plusieurs maladies à la plante (Gardner et al., 2015). En outre, l'activité de ponte des Tephritidae sur les fruits est source de nombreux dégâts variant de 30 à 100\% de la production (Dhillon et al., 2005).

En vue d'augmenter leurs rendements, les maraîchers du département de Korhogo font recours à l'utilisation massive de pesticides (Tuo et al., 2017 ; Koné et al., 2019b). Cependant, de nombreux travaux ont montré que les doses d'application recommandées ne sont pas respectées et seulement $27 \%$ des pesticides utilisés par les maraîchers sont homologués (Doumbia et Kwadjo, 2009 ; Tuo et al., 2017 ; Koné et al., 2019b). L'utilisation abusive et non réglementée de ces pesticides, favorise le développement de résistances chez les insectes ravageurs (Zhou et al., 2011), l'élimination des ennemis naturels de ces ravageurs (Smyth et Hoffman 2010), mais aussi à celle des insectes pollinisateurs (Frazier et al., 2015).

Dans le but de trouver d'autres produits alternatifs, non nocifs à l'environnement, compatibles avec la gestion intégrée des ravageurs, la présente étude a été menée dans le département de Korhogo. Cette étude a consisté à comparer l'efficacité des extraits aqueux, hydro-alcoolique et des huiles essentielles de $\mathrm{O}$. canum à celle d'un insecticide de synthèse homologué en cultures maraichères.

\section{Matériel et Méthodes}

\section{Zone d'étude}

L'étude a été conduite en 2017 pendant la saison pluvieuse et la saison sèche sur une parcelle expérimentale logée au sein du carré potager de l'Université Peleforo Gon Coulibaly de Korhogo. La sous-préfecture de Korhogo est située entre 8o26 et 10o27 de latitude Nord et 5o17 et 6o19 de longitude Ouest à $600 \mathrm{Km}$ d'Abidjan dans le Nord de la Côte-d'Ivoire.

La localité appartient au régime climatique tropical sec de type soudano-Sahélien dont le rythme des saisons est réglé par le déplacement du Front Intertropical (Jourda et al., 2005). Ce climat est caractérisé par deux saisons. La saison des pluies qui s'étend de Mai à Octobre avec un maximum 
de précipitation en septembre et la saison sèche de Novembre à Avril, caractérisée par l'harmattan qui s'installe de Décembre à Février. La pluviométrie moyenne annuelle varie entre 1100 et $1600 \mathrm{~mm}$ et la température moyenne annuelle varie entre 25 et $35^{\circ} \mathrm{C}$ (Kouakou et al., 2014).

\section{Evaluation de l'efficacité d'extraits de plante sur les insectes ravageurs Dispositif expérimental}

Le dispositif utilisé était un bloc de Fisher complètement randomisé avec cinq (5) objets (traitements) et cinq (5) répétitions (blocs).

Chaque parcelle élémentaire mesurait $1 \mathrm{~m} 2(1 \mathrm{~m} \mathrm{x} 1 \mathrm{~m})$ et toutes les parcelles étaient séparées l'une de l'autre par une allée de $1 \mathrm{~m}$ de large. Avant le semis, un labour à la main suivi du nivellement, du piquetage et de l'apport de fiente de poulet dans chaque poquet a été effectué. Le semis a été effectué, à raison de trois graines par poquets. Deux jours après la levée, un démariage a été effectué de sorte à conserver 2 plants par poquet, soit huit (8) plants par parcelle élémentaire et un total de 200 plants pour toute la parcelle expérimentale.

\section{Préparation des extraits aqueux et hydro-alcooliques de feuilles de $\mathbf{O}$. canum}

Les plantes de O. canum ont été collectées dans les villages de Nangakaha et Fonofla dans la sous-préfecture de Korhogo. Pour la préparation des extraits, les feuilles fraiches de $\mathrm{O}$. canum récoltées ont été aussitôt lavées puis broyées dans un mortier jusqu'à l'obtention d'une pâte. Cinq (5) Kg de feuilles fraiches ont permis d'obtenir un (1) Kg de pâte. Pour l'extrait aqueux, la pâte de feuilles a été introduite dans un seau contenant de l'eau à la dose de 1 kilogramme pour 15 litres d'eau (67g/l). L'extrait hydroalcoolique a été obtenu par dilution de la même masse de feuilles écrasée dans une solution d'alcool à $10 \%$ à la dose de $67 \mathrm{~g} / \mathrm{L}$. Chaque mélange a été remué énergiquement toutes les deux heures. Après 24 heures de macération pour l'extrait hydro-alcoolique et trois jours pour l'extrait aqueux (Diabaté et al. 2014). Chacune des solutions a été filtrée au travers d'un papier filtre Whatman. Les filtrats obtenus ont été conservés dans des fûts en plastique de 20 litres étiquetés et fermés hermétiquement. Ces filtrats ont servi pour les différents traitements des parcelles.

\section{Extraction des huiles essentielles de 0 . canum}

Cinq (5) $\mathrm{Kg}$ de feuilles de $\mathrm{O}$. canum séchées à l'ombre, ont été mélangées dans 15 litres d'eau et portés à ébullition dans une barrique de contenance 50 litres. Cette dernière était surmontée d'un tuyau en cuivre transportant les vapeurs d'eau et allant se plonger dans une deuxième barrique pleine d'eau servant de bassin de refroidissement des vapeurs. A la sortie du 
bassin de refroidissement, il est recueilli dans un bocal en verre un distillat composé de l'eau florale et de l'huile essentielle $(333 \mathrm{~g} / \mathrm{L})$. Le distillat est versé dans une ampoule à décanter afin de séparer l'huile essentielle et l'eau florale.

\section{Application des extraits sur les plants}

Quatre (4) pulvérisateurs à main, de marque JARDINOVA d'une contenance d'un litre chacun ont été utilisés. Chaque pulvérisateur a servi à l'application d'un seul biopesticide afin d'éviter les contaminations. Lors du traitement, selon la méthode utilisée par Gnago et al. (2010) chaque parcelle élémentaire a été isolée par un plastique en polyéthylène pour empêcher que le produit pulvérisé atteigne la parcelle élémentaire contiguë. Pour que le traitement soit homogène et dans le but d'atteindre une population importante de ravageurs, les jets ont été orientés de sorte à recouvrir les faces inférieures et supérieures des feuilles de chaque plante. Les premières applications foliaires ont débuté dès la germination des plants.

Les traitements étaient hebdomadaires avec six (6) jours d'intervalle (Gnago et al., 2010 ; Diabaté et al., 2014). Les traitements appliqués sur les parcelles de courgette étaient l'extrait aqueux de feuilles de O. canum 67g/L (T1), l'extrait hydro-alcoolique de feuilles de O. canum $67 \mathrm{~g} / \mathrm{L}$ (T2), l'huile essentielle des feuilles de O. canum $333 \mathrm{~g} / \mathrm{L}$ (T3) et l'insecticide de synthèse Cypercal 50g/L (T4), insecticide de référence en culture maraichère. L'huile essentielle était diluée dans de l'eau additionnée à $10 \mathrm{ml}$ de savon liquide pour assurer la miscibilité de l'eau et de l'huile. Les parcelles témoins (T0) n'ont pas été traitées.

\section{Evaluation de l'efficacité des extraits de $O$. canum sur les insectes ravageurs}

L'évaluation de l'efficacité des extraits de plante sur les insectes ravageurs a consisté à dénombrer le nombre d'insectes par espèces en fonction des traitements Elle était réalisée le matin entre $6 \mathrm{~h}$ et $8 \mathrm{~h}$, trois jours après chaque traitement (Diabaté et al., 2014). Les observations ont été effectuées sur la totalité des huit plants de chaque parcelle élémentaire.

\section{Evaluation de l'efficacité des extraits de plante dans la réduction des taux d'attaque}

Elle a consisté déterminer le nombre de feuilles, de fleurs et de fruits attaqués en fonction du traitement par rapport au nombre total de chacun de ces organes. Elle était réalisée une fois par semaine, sur tout le cycle de production. 


\section{Analyses statistiques}

Toutes les données ont été traitées à l'aide du logiciel Statistica, version 7.1. Dans la présente étude, des analyses de variances à un facteur (ANOVA, $\mathrm{p}<0,05)$ ont été réalisées puis les moyennes homogènes ont été regroupées à l'aide des tests de Newman-Keuls au seuil de 5\%.

\section{Résultats \\ Efficacité des extraits de $O$. canum dans la réduction du nombre de pucerons}

Au cours de la phase végétative, le nombre de pucerons récolté sur la parcelle témoin était en moyenne de $10 \pm 2,1$ par plant. Pour le traitement avec l'extrait aqueux, il a été récolté $5 \pm 1,66$ pucerons, $4 \pm 1,63$ pour l'extrait hydro-alcoolique, $5 \pm 1,66$ pour l'huile essentielle et de $1 \pm 1$ pour l'insecticide de synthèse. Les analyses de variances ont révélé une différence significative $(\mathrm{F}=3,84 ; \mathrm{p}<0,05)$ entre l'efficacité des différents produits utilisés comparés au témoin. Les différents extraits de la plante ont présenté une efficacité moyenne par rapport au témoin et à l'insecticide de synthèse (Figure 1).

Pendant la floraison, les moyennes de pucerons récoltés après traitement étaient respectivement de $8 \pm 4,89$ pour l'extrait aqueux, $21 \pm 6,04$ pour l'huile essentielle et l'extrait hydro-alcoolique, $23 \pm 5,38$ (Figure 1). Comparé au témoin $(62 \pm 4,66)$, l'insecticide de synthèse n'a eu aucune efficacité dans la réduction du nombre moyen de pucerons par plant $(56 \pm 7,18)$. Les analyses statistiques ont révélé une différence significative entre l'efficacité de ces différents produits $(\mathrm{F}=17,144 ; \mathrm{p}<0,05)$.

Le test de Newman-Keuls a fait ressortir deux groupes homogènes. L'extrait aqueux, l'huile essentielle et l'extrait hydro-alcoolique ont constitué le premier groupe homogène. Le second groupe homogène était constitué de l'insecticide de synthèse et du témoin .

$\mathrm{Au}$ cours de la phase de floraison-fructification, les analyses statistiques ont révélé une différence significative $(F=36,141 ; p<0,05)$ entre l'efficacité des différents produits testés (Figure 1). Le test de séparation de Newman-Keuls laisse apparaitre trois groupes homogènes. Le premier groupe homogène est constitué de l'extrait aqueux. Avec un nombre moyen résiduel de $17 \pm 2,13$ pucerons par plant, l'extrait aqueux a été le produit qui a le mieux contrôlé les pucerons. Le deuxième groupe homogène est composé de l'extrait hydro-alcoolique et l'huile essentielle. Le nombre moyen de pucerons par plant pour ces produits a été respectivement de $43 \pm 6,67$ et de $53 \pm 9,55$. Concernant le troisième groupe homogène, il est constitué de l'insecticide de synthèse $(103 \pm 7,89)$ et du témoin $(114 \pm 5,61)$. 


\section{Efficacité des extraits de $O$. canum dans la réduction du nombre d'aleurodes}

Au niveau de la phase végétative, les analyses statistiques ont révélé une différence significative entre l'efficacité des différents produits utilisés pour lutter contre ces insectes $(F=19,599$; $p<0,05)$. Le test de NewmanKeuls a montré une réduction élevée du nombre moyen de mouches blanches par plant avec l'extrait aqueux $(25 \pm 7,63)$, l'extrait hydro-alcoolique (30 $\pm 4,47)$ et l'huile essentielle $(30 \pm 7,3)$. L'insecticide de synthèse n'a eu aucun effet significatif comparé au témoin. Le nombre moyen résiduel de mouches blanches par plant a été de $72 \pm 4,49$ pour l'insecticide de synthèse et de $74 \pm$ 4 pour le témoin (Figure 2).

Durant la floraison, le nombre d'aleurodes récoltés après chaque traitement a varié en fonction du produit utilisé (Figure 2). Ainsi, pour l'extrait hydro-alcoolique, il était de $15 \pm 2,23 ; 18 \pm 4,16$ pour l'extrait hydro alcoolique et de $18 \pm 4,16$ pour l'extrait aqueux. Ces produits ont été les plus efficaces contre les mouches blanches. L'huile essentielle $(30 \pm 6,32)$ et l'insecticide de synthèse $(42 \pm 6,96)$ ont présenté une efficacité intermédiaire. Au niveau du témoin le nombre moyen de mouches blanches par plant a été de $62 \pm 3,59$ (Figure 2). L'analyse de variance ANOVA $(F=14,951 ; p<0,05)$ suivi du test Newman-Keuls a révélé trois groupes homogènes. Le premier groupe homogène est constitué par l'extrait hydro -alcoolique et l'extrait aqueux. Le deuxième groupe homogène est formé par l'huile essentielle et l'insecticide de synthèse. Le témoin a constitué le troisième groupe ho mogène. $\mathrm{Au}$ cours de la phase de floraison-fructification, l'ensemble des produits utilisés contre les aleurodes ont été plus efficaces que le témoin (Figure 2). Les analyses de la variance ont révélé une différence significative ( $F=22,026$ $; \mathrm{p}<0,05)$ entre l'efficacité des différents produits utilisés. Le test de Newman-Keuls a montré quatre groupes homogènes. L'extrait hydroalcoolique $(14 \pm 1,63)$ et l'extrait aqueux $(17 \pm 2,13)$ ont réduit significativement le nombre de mouches blanches par rapport au témoin. Avec en moyenne $33 \pm 6,5$ aleurodes par plant, l'insecticide de synthèse a montré une efficacité moyenne. Le troisième groupe homogène est constitué par l'huile essentielle. Pour l'huile essentielle, le nombre moyen résiduel de mouches blanches par plant a été de $46 \pm 4,76$. Le témoin a présenté la moyenne de pucerons par plants la plus élevée $(62 \pm 4,42)$.

\section{Efficacité des extraits de $O$. canum dans la réduction du nombre de Chrysomèles}

Durant la phase végétative de la courgette, la réduction du nombre de Chrysomelidae par rapport au témoin a varié significativement en fonction du traitement $(\mathrm{F}=3,75 ; \mathrm{p}<0,05)$. Le test de séparation de Newman-Keuls a 
révélé trois groupes homogènes en fonction de l'efficacité du traitement (Figure 3).

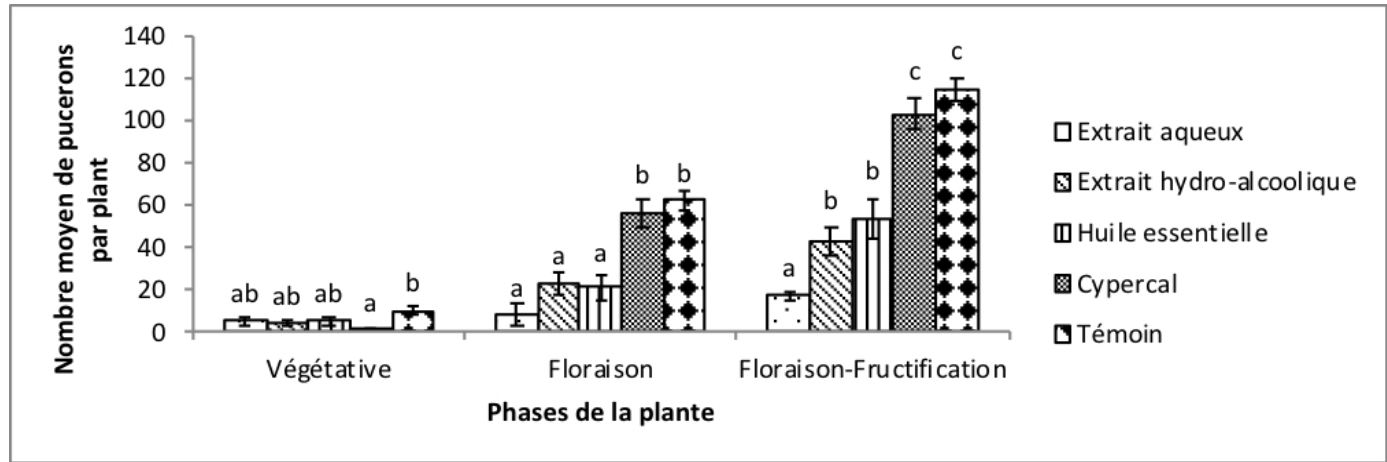

Figure 1 : Efficacité des extraits de O. canum dans la réduction du nombre de pucerons

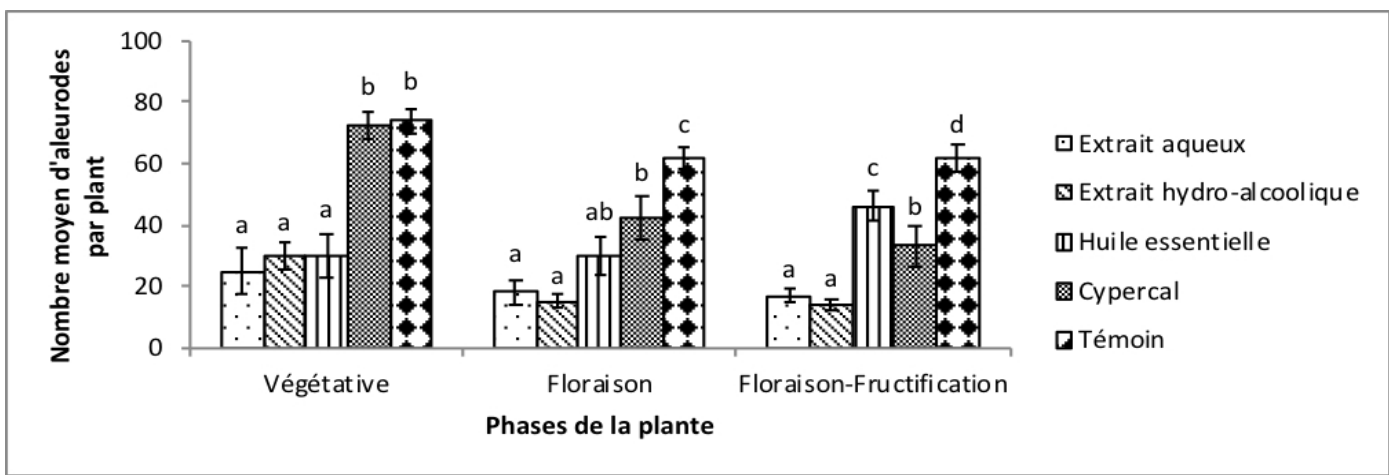

Figure 2 : Efficacité des extraits de O. canum dans la réduction du nombre d'aleurodes

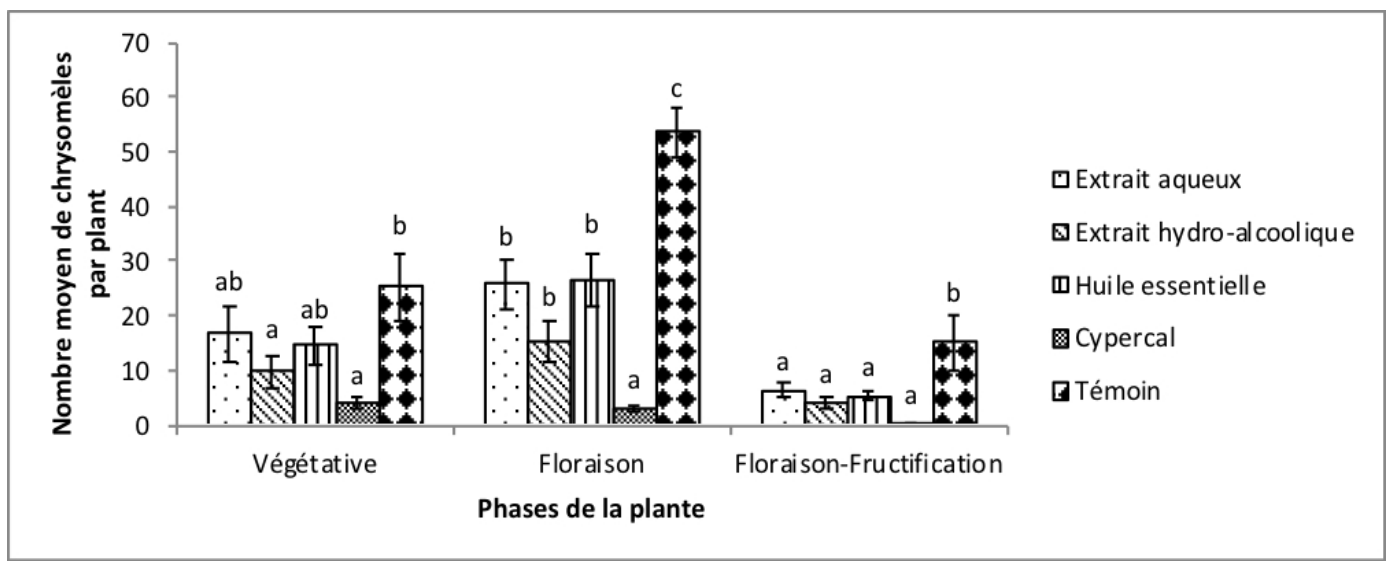

Figure 3 : Efficacité des extraits de O. canum dans la réduction du nombre de Chrysomèles Pour une phase donnée, les barres affectées de lettres différentes sont significativement différentes au seuil de $5 \%$ selon le test de Newman-Keuls.

Le premier groupe était constitué du Cypercal et de l'extrait hydroalcoolique. Ces produits ont présenté la plus grande réduction significative des ravageurs . Pour ces produits, l'abondance moyenne des chrysomèles était 
respectivement de $4 \pm 1,15$ et de $9,8 \pm 2,82$ par plant. Pour l'huile essentielle $(14,6 \pm 3,36)$ et l'extrait aqueux $(16,7 \pm 4,95)$ la réduction du nombre de chrysomèles était moyenne par rapport au témoin (Figure 3).

Pendant la floraison de la courgette, l'insecticide de synthèse et les différents extraits ont eu un impact significatif dans la réduction du nombre de chrysomèles par rapport au témoin $(\mathrm{F}=22,4 ; \mathrm{p}<0,05)$. Avec une abondance moyenne de 3,1 $\pm 0,7$ chrysomèles par plant, l'insecticide de synthèse a présenté l'efficacité la plus élevée. L'extrait hydro-alcoolique $(15,2 \pm 3,59)$, l'extrait aqueux $(25,8 \pm 4,35)$ et l'huile essentielle $(26,6 \pm 4,98)$ ont montré une efficacité moyenne dans la réduction du nombre de ravageurs (Figure 3).

Concernant la phase de floraison-fructification, les analyses statistiques ont révélé une différence significative entre l'efficacité des produits insecticides par rapport au témoin $(\mathrm{F}=5,05 ; \mathrm{p}<0,05)$. Le test de séparation de Newman-Keuls a scindé les produits utilisés en deux groupes homogènes. Le premier groupe constitué de l'insecticide de synthèse $(0,3 \pm$ $0,15)$, l'extrait hydro-alcoolique $(4,1 \pm 0,95)$, l'huile essentielle $(5,4 \pm 0,9)$ et l'extrait aqueux $(6,4 \pm 1,23)$ ont présenté une efficacité similaire. Le deuxième groupe homogène est constitué par le témoin (Figure 3).

\section{Efficacité des extraits de 0 . canum dans la réduction du taux d'attaque des piqueurs -suceurs}

$\mathrm{Au}$ niveau des feuilles, les analyses statistiques n'ont révélé aucune différence significative entre les taux d'attaque moyens obtenus avec les différents produits $(\mathrm{F}=0,926 ; \mathrm{p}>0,05)$. Pour chacun des produits testés, le taux d'attaque moyen des feuilles a été inférieur à $10 \%$. Ces attaques de feuilles étaient visibles uniquement pendant la phase végétative et pendant la phase de floraison. Aucune attaque due aux pucerons et aux aleurodes n'a été enregistrée pendant la phase de floraison-fructification (Tableau I).

Concernant les fleurs, L'ensemble des dégâts dus aux pucerons et aux aleurodes ont été recensés uniquement au niveau des fleurs mâles. Aucun dégât n'a été recensé sur les fleurs femelles de la courgette durant tout le cycle. Pour les différents produits testés, les analyses de variance ont montré une différence significative entre l'efficacité de ces produits $(\mathrm{F}=15,422 ; \mathrm{p}<0,05)$. Selon le test de séparation de Newman-Keuls, les produits testés se classent en trois groupes homogènes. Le premier groupe homogène est formé par l'extrait hydro-alcoolique et l'extrait aqueux. Ces deux extraits ont présenté les taux moyen de fleurs attaquées les plus faibles. Pour l'huile essentielle et l'insecticide de synthèse, les fleurs étaient moyennement attaquées. Ces deux produits ont constitué le deuxième groupe homogène. Présentant le taux d'attaque le plus élevé, le témoin a constitué le troisième groupe homogène (Tableau I). 
Efficacité des extraits de $\mathrm{O}$. canum dans la réduction des attaques des Chrysomèles et des mouches de la courgette

$\mathrm{Au}$ niveau des feuilles, les taux d'attaque ont varié significativement en fonction du produit utilisé $(\mathrm{F}=56,13 ; \mathrm{p}<0,05)$. Ainsi, il était de $24 \pm 1 \%$ pour le cypercal, $48 \pm 2 \%$ pour l'extrait hydro-alcoolique et $57 \pm 3 \%$ pour l'huile essentielle. Concernant le témoin, la moyenne était de $80 \pm 2 \%$ par plant (Figure 4).

Tableau I : Efficacité des extraits de O. canum dans la réduction des dégâts des piqueurssuceurs

\begin{tabular}{lcc}
\hline \multicolumn{1}{c}{ Traitement } & Taux d'attaque feuilles & Taux d'attaque fleurs \\
\hline Témoin & $0,44 \pm 0,05^{\mathrm{a}}$ & $0,043 \pm 0,005^{\mathrm{c}}$ \\
Extrait aqueux & $0,07 \pm 0,02^{\mathrm{a}}$ & $0,01 \pm 0,002^{\mathrm{a}}$ \\
Extrait hydro-alcoolique & $0,019 \pm 0,03^{\mathrm{a}}$ & $0,008 \pm 0,003^{\mathrm{a}}$ \\
Huile essentielle & $0,067 \pm 0,002^{\mathrm{a}}$ & $0,024 \pm 0,002^{\mathrm{b}}$ \\
Insecticide de synthèse & $0,057 \pm 0,004^{\mathrm{a}}$ & $0,032 \pm 0,004 \mathrm{~b}^{\mathrm{c}}$ \\
F & 0,926000 & 15,422 \\
Ddl & 4 & 4 \\
P & $\mathrm{p}>0,05$ & $\mathrm{p}<0,05$ \\
\hline
\end{tabular}

Dans une colonne, les moyennes affectées de lettres différentes sont significativement différentes au seuil de 5\% selon le test de Newman-Keuls

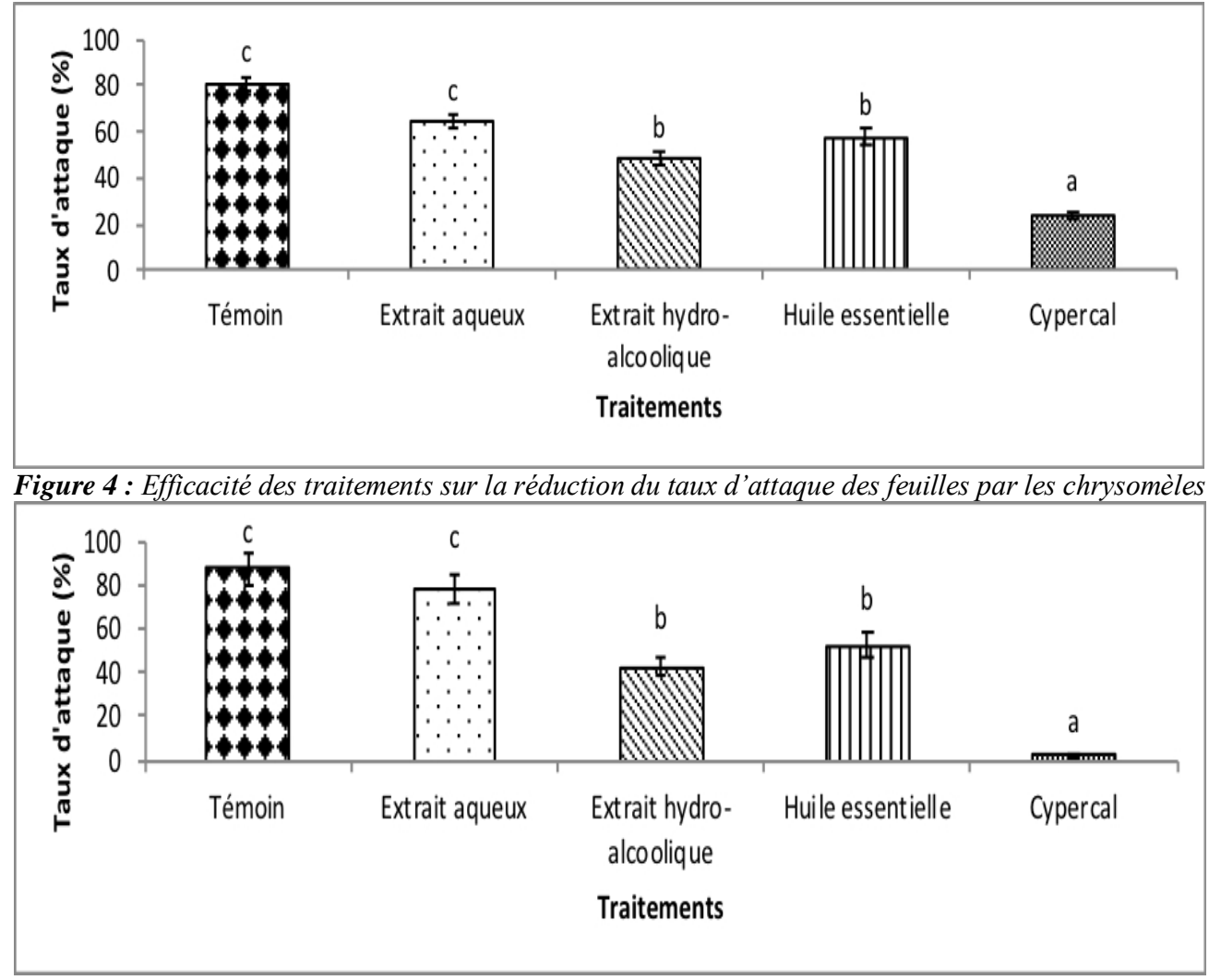

Figure 5 : Efficacité des traitements sur la réduction du taux d'attaque des fleurs par les chrysomèles 
Les barres affectées de lettres différentes sont significativement diférentes au seuil de 5\% selon le test de Newman-Keuls.

Concernant les fleurs, excepté l'extrait aqueux, l'utilisation de l'insecticide de synthèse, l'extrait hydro-alcoolique et l'huile essentielle pour protéger les fleurs de courgette contre les chrysomèles ont produit des résultats significativement supérieurs à ceux obtenus avec le témoin $(\mathrm{F}=11,07 ; \mathrm{p}<$ 0,05). Le produit ayant réduit le plus les attaques était le Cypercal avec seulement $2,1 \pm 1,1 \%$ de fleurs par plant présentant des signes d'attaque. Il était secondé par l'extrait hydro-alcoolique et l'huile essentielle, avec respectivement des moyennes de $42,4 \pm 3,8 \%$ et $52,3 \pm 5,9 \%$. Le taux d'attaque enregistré sur les parcelles traitées avec l'extrait aqueux $(78,1 \pm$ $6,3 \%)$ n'était pas significativement différent de celui obtenu avec le témoin $(87,1 \pm 6,9 \%)$ (Figure 5).

Les attaques sur les fruits ont été causées par les chrysomèles et par les mouches de la courgette. La réduction du taux d'attaque a varié en fonction du ravageur et du type d'insecticide.

Concernant les chrysomèles (Figure 6), la moyenne la plus élevée de fruits attaqués a été observée avec l'extrait aqueux $(35,9 \pm 10,1 \%)$ et le témoin $(28 \pm 11,9 \%)$. Les taux d'attaque les plus faibles ont été obtenus respectivement avec l'huile essentielle $(8,5 \pm 4,1 \%)$, l'extrait hydro-alcoolique $(5,1 \pm 2,7 \%)$ et l'insecticide de synthèse $(0,9 \pm 0,1 \%)$. Les analyses de variance $(\mathrm{F}=5,12 ; \mathrm{p}<0,05)$ ont révélé une différence significative entre l'efficacité de ces traitements.

S'agissant des mouches, les attaques les plus importantes ont été observées sur les parcelles traités avec l'huile essentielle, l'insecticide de synthèse, et l'extrait hydro-alcoolique. Pour ces produits, la proportion moyenne de fruits attaqués était respectivement de 68,3 $\pm 9, \%, 67,9 \pm 5,1 \%$, et $56,3 \pm 10,2 \%$. Les attaques les plus faibles ont été enregistrées avec le témoin $(48,4 \pm 13,9 \%)$ et l'extrait aqueux de $O$. canum $(44,4 \pm 11,1 \%)$. Toutefois les analyses statistiques n'ont révélé aucune différence significative entre ces taux $(p>0,05)$ (Figure 7).

S'agissant du nombre moyen de fruits sains par plant, il a varié significativement en fonction du traitement $(\mathrm{F}=7,41 ; \mathrm{p}<0,05)$. Le nombre moyen de fruits sains par plant le plus élevé a été obtenu avec l'insecticide de synthèse $(1,4 \pm 0,21)$. Par contre les différentes moyennes obtenue avec l'extrait hydro-alcoolique $(0,8 \pm 0,17$ fruits/plant $)$, l'huile essentielle $(0,53 \pm$ 0,21 fruits/plant), et l'extrait aqueux $(0,26 \pm 0,11$ fruits/plant $)$ étaient statistiquement semblables (Figure 8). 


\section{Impact des extraits de $\boldsymbol{O}$. canum sur le nombre et la masse des fruits.}

S'agissant des fruits, le nombre moyen a varié en fonction du traitement insecticide. Les analyses de variance ont révélé une différence significative entre les différents traitements $(\mathrm{F}=4,523 ; \mathrm{p}<0,05)$. Le test de Newman-Keuls a permis de classer les produits testés en deux groupes homogènes. Avec un nombre moyen de fruits par pied de 5,20 $\pm 0,64$, l'huile essentielle a donné le rendement le plus élevé. L'extrait hydro-alcoolique a présenté un rendement moyen de 4,6 $\pm 0,49$ fruits par plant. Cet extrait a été le second produit le plus efficace. Les rendements moyens obtenus avec l'extrait aqueux $(3,2 \pm 0,24)$, le témoin $(3,4 \pm 0,44)$ et l'insecticide de synthèse $(3,4 \pm 0,16)$ sont similaires (Figure 9$)$ et ont été les plus faibles. Concernant le poids moyen des fruits, il est significativement plus élevé pour tous les traitements par rapport au témoin $(\mathrm{F}=4,483 ; \mathrm{p}<0,05)$. Cependant aucune différence significative n'a été obtenue entre le poids moyen par fruit obtenu avec l'extrait aqueux $(0,9 \pm 0,05)$, l'insecticide de synthèse $(0,91 \pm 0,05)$, l'extrait hydro-alcoolique $(0,951 \pm 0,1)$ et l'huile essentielle dont la moyenne était de $0,952 \pm 0,13$ (Figure 10).

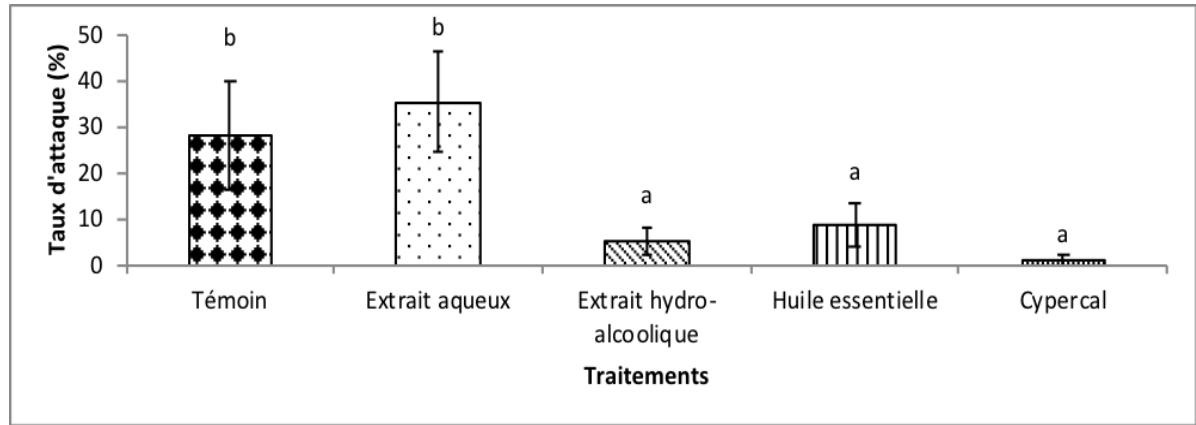

Figure 6: Efficacité des traitements sur la réduction du taux d'attaque des fruits par les chrysomèles

Les barres affectées de lettres différentes sont significativement différentes au seuil de 5\% selon le test de Newman-Keuls.

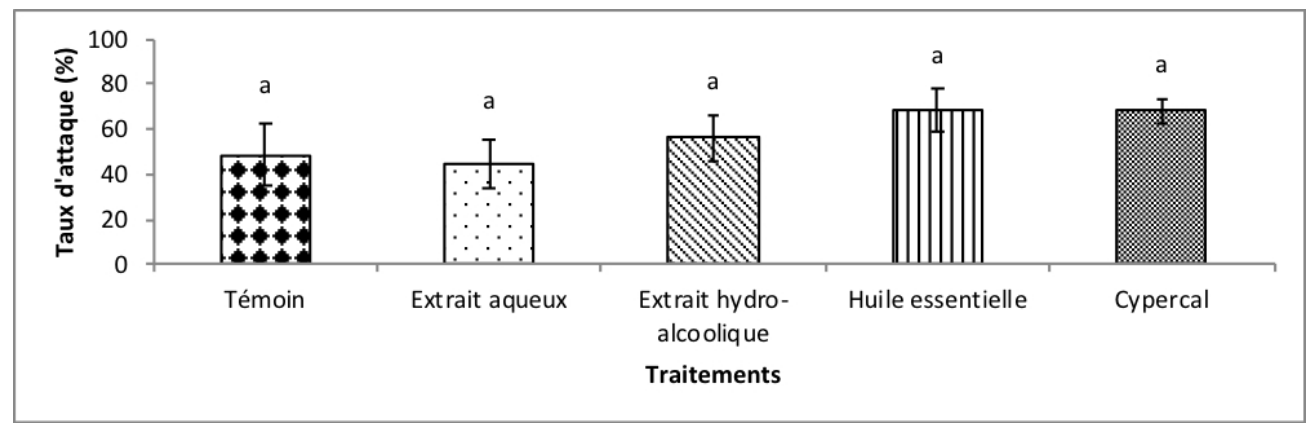

Figure 7 : Efficacité des traitements sur la réduction du taux d'attaque des fruits par les Mouches de la courgette 
Les barres affectées de lettres différentes sont significativement différentes au seuil de 5\% selon le test de Newman-Keuls.

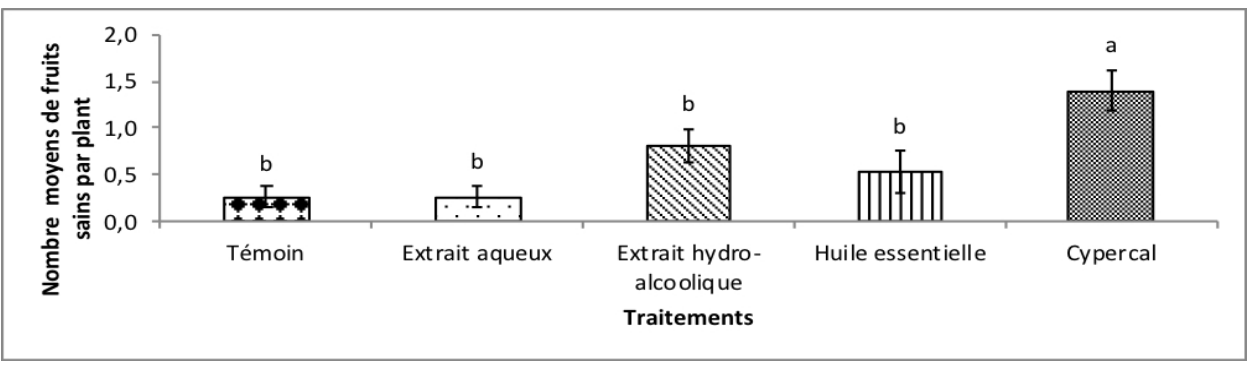

Figure 8 : Efficacité des traitements sur la qualité des fruits

Les barres affectées de lettres différentes sont significativement différentes au seuil de 5\% selon le test de Newman-Keuls.

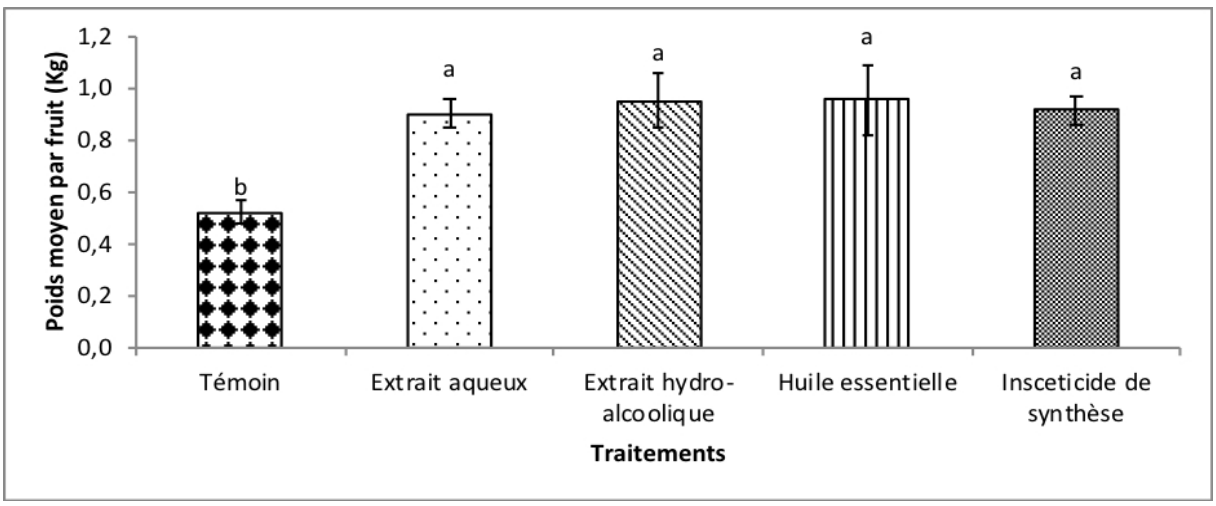

Figure 9 : Nombre moyen de fruits sains par plant en fonction du traitement

Les barres affectées de lettres différentes sont significativement différentes au seuil de 5\% selon le test de Newman-Keuls.

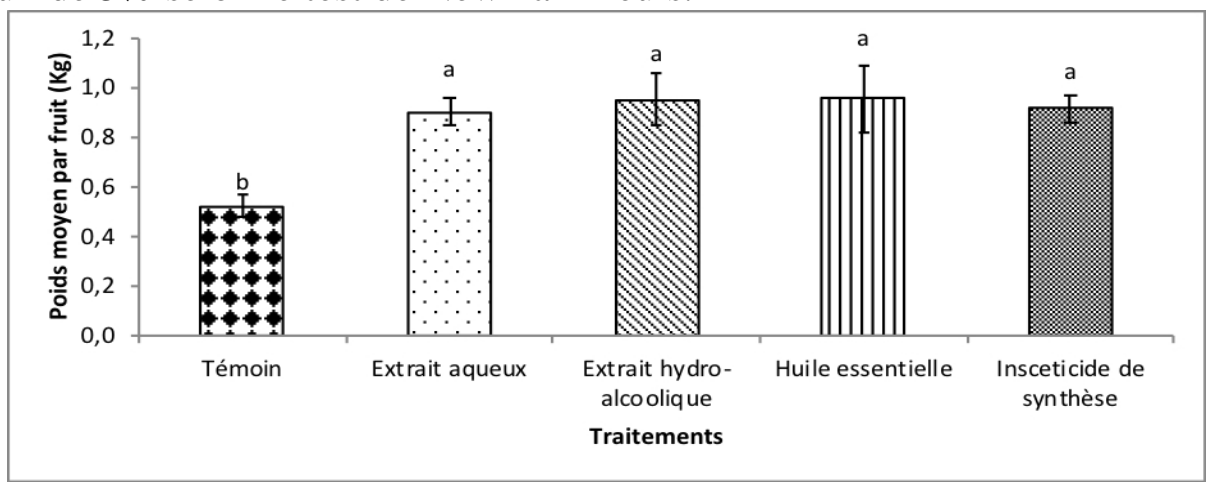

Figure 10 : Poids moyen par fruit en fonction du traitement

Les barres affectées de lettres différentes sont significativement différentes au seuil de 5\% selon le test de Newman-Keuls. 


\section{Discussion}

Les analyses ont montré que les extraits de plante ont été efficaces dans le contrôle des pucerons, des aleurodes et des chrysomèles. Cette efficacité serait liée aux propriétés insecticides des composés de cette plante. Ces résultats sont proches de ceux de Bassole et al. (2003). Ces auteurs ont montré que les huiles essentielles provenant des feuilles de $O$. canum ont des propriétés insecticides et bactéricides. Les principaux composés des feuilles et des fleurs de $O$. canum sont le 1,8-cineole (60.1\%) et le cis, trans-piperitol (68.5\%) (Tonzibo et al., 2008). Ces résultats sont proches de ceux de Jacobson (1982) ; Stevenson et al. (2009). Pour ces auteurs, certaines plantes contiennent des agents bioactifs tels que les composés phénoliques, les alcaloïdes, les tanins, les flavonoïdes, les stéroïdes et les triterpènes qui ont des propriétés insecticides. Selon Gopalakrishnan et al. (2005) ces composés auraient également des propriétés répulsives, toxiques, dissuasives et inhibitrices de la reproduction des insectes. Des observations similaires ont été faites par Savadogo et al. (2016) au Burkina Faso. Ces auteurs ont mis en évidence les caractéristiques insecticides et insectifuges de $O$. canum. En effet, les feuilles de cette plante se sont révélées efficaces dans la lutte contre les moustiques et pour la conservation des semences stockées. Les travaux de Kobenan et al. (2018) ont également démontré le potentiel insecticide des huiles essentielles de $O$. canum sur la chenille Pectinophora gossypiella en conditions de laboratoire.

Il est également ressorti des analyses, une efficacité des extraits de plante dans la réduction des attaques occasionnées par les pucerons, les aleurodes et les chrysomèles. Cette efficacité observée est due au fait que ces différents extraits utilisés ont des principes actifs efficace sur les ravageurs. Ces résultats sont semblables à ceux obtenus par Ilboudo et al. (2015). Ces auteurs ont rapporté que l'huile essentielle de $O$. canum a permis de protéger les denrées stockées de niébé contre les attaques de Callosobrus maculatus.

Les biopesticides utilisés n'ont pas été efficace sur les mouches. Cela pourrait être lié à la biologie de ces insectes. En effet, selon Tuo et al. (2018), les mouches passent très peu de temps sur les plantes de courgette. Elles viennent juste sur les fruits pour pondre puis elles retournent s'abriter sur les plantes refuges. Elles échapperaient ainsi à l'action des pesticides. Selon Dhillon et al. (2005); Bonnet et al. (2010), les mouches femelles passent plus de temps sur les plantes refuges que sur les Cucurbitaceae. Ce comportement rend difficile la lutte étant donné que les adultes ne s'alimentent pas sur la courgette. La difficulté de la lutte contre les mouches de la courgette est liée également au mode de développement de ces insectes. Les femelles pondent leurs œufs dans les fruits tendres en perçant le fruit avec l'ovipositeur. A l'éclosion, les larves se nourrissent de la pulpe, entrainant ainsi la pourriture du fruit (Dhillon et al., 2005 ; Ganie et al., 2012). 


\section{Conclusion}

Au terme de cette étude, il ressort que les différents extraits de $O$. canum sont efficaces dans le contrôle des principaux ravageurs de la courgette et dans la réduction de leurs dégâts, à l'exception des mouches des fruits. L'extrait hydro-alcoolique $(67 \mathrm{~g} / \mathrm{L})$ et l'extrait aqueux $(67 \mathrm{~g} / \mathrm{L})$, ont été les plus efficaces sur les pucerons et les aleurodes. Au niveau des chrysomèles, l'extrait hydro-alcoolique (67 g/L) et l'huile essentielle (333 g/L) étaient les plus efficaces. Ces produits pourraient constituer une alternative à l'usage des pesticides de synthèse dans la gestion des ravageurs de la courgette au nord de la Côte d'Ivoire compte tenu de la disponibilité de la plante en saison des pluies et la non complexité de la méthode d'extraction. Il convient également de vérifier l'efficacité des extraits de $O$. canum sur les mouches des fruits en traitant les plantes refuges aux alentours des parcelles de courgette pendant les différents traitements.

\section{References:}

1. Bassole, I.H.N., Guelbeogo, W.M., Nébié R., Costantini, C., Sagnon, N.F., Kabore Z.I., \& Traoré, S.A. (2003). Ovicidal and larvicidal activity against Aedes aegypti and Anopheles gambiae complex mosquitoes of essential oils extracted from three spontaneous plants of Burkina Faso. Parassitologia, 45(1), 23-26.

2. Bonnet, E. (2010). Interactions entre les mouches des Cucurbitaceae (Diptera: Tephritidae), une plante hôte (courgette) et une plante piège (maïs) disposée en bandes et patchs intra-parcellaires à la Réunion (Doctoral dissertation, Université de la Réunion).

3. Dhillon, M.K., Singh, R., Naresh, J.S., \& Sharma, H.C. (2005). The melon fruit fly, Bactrocera cucurbitae: A review of its biology and management. Journal of Insect Science, 5(1), 40. https://doi.org/10.1093/jis/5.1.40

4. Diabaté, D., Gnago, J.A., Koffi, K., \& Tano, Y. (2014). The effect of pesticides \& aqueous extracts of Azadirachta indica (A.juss) and Jatropha carcus L. on Bemisia tabaci (Gennadius) and Helicoverpa armigera (Hübner) found on tomato plants in Cote d'Ivoire. Journal of Applied Biosciences, 80(1), $7132 \quad$ - 7143. http://dx.doi.org/10.4314/jab.v80i1.14

5. Doumbia, M., \& Kwadjo, K.E. (2009). Pratiques d'utilisation et de gestion des pesticides par les maraîchers en Côte d'Ivoire : Cas de la ville d'Abidjan et deux de ses banlieues (Dabou et Anyama). J. Appl. Biosci., 18, 992-1002. Published at www.biosciences.elewa.org.

6. Frazier, M.T., Leslie, T.W., Mullin, C.A., Mussen, E.C., Frazier, J.L., Ashcraft, S.A., \& Drummond, F.A. (2015). Assessing Honey Bee (Hymenoptera: Apidae) Foraging Populations and the Potential Impact 
of Pesticides on Eight U.S. Crops. J. Econ. Entomol., 108 (5), $2141-$ 2152. https://doi.org/10.1093/jee/tov195

7. Ganie, S.A., Khan, Z.H., Ahangar, R.A., Bhat, H.A., \& Barkat, H. (2012). Population Dynamics, Distribution, and Species Diversity of Fruit Flies on Cucurbits in Kashmir Valley, India. Journal of Insect Science, 13(1), 1-7. https://doi.org/10.1673/031.013.6501

8. Gardner, J., Hoffmann, M.P., \& Mazourek M. (2015). Striped Cucumber Beetle (Coleoptera: Chrysomelidae) Aggregation in Response to Cultivar and Flowering. Environ. Entomol., 44 (2), 309316. http://www.insectscience.org/13.65

9. Gnago, J.A., Danho, M., Agneroh, A.T., Fofana, I.K., \& Kohou, A.G. (2010). Efficacité des extraits de neem (Azadirachta indica) et de papayer (Carica papaya) dans la lutte contre les insectes ravageurs du gombo (Abelmoschus esculentus) et du chou (Brassica oleracea) en Côte d'Ivoire. International Journal of Biological and Chemical. Sciences, 4 (4), 953-966. http://dx.doi.org/10.4314/ijbcs.v4i4.63035

10. Gopalakrishnan, S., \& Martin, Rathi J. (2005). Insecticidal activity of aerial parts of Synedrella nodiflora Gaertn (Compositae) on Spodoptera litura (FAB.). Journal of Central European Agriculture, 6(3), 223-228. https://doi.org/10.5513/jcea.v6i3.292

11. Ilboudo, Z., Dabiré-Binso, C.L., Sankara, F., Nébié, R.C.H., \& Sanon, A. (2015). Optimizing the Use of Essential Oils to Protect Stored Cowpeas from Callosobruchus maculatus (Coleoptera: Bruchinae) Damage. African Entomology, 23(1), 94-100. https://hdl.handle.net/10520/EJC167521

12. Jacobson, M. (1982). Plants, insects, and man-their interrelationships. Economic Botany, 36(3), 346-354. https://doi.org/10.1007/BF02858560

13. Jourda, J.P, Saley, B.M., Djagoua, E.V., Kouamé, K.J., Biémi, J., \& Razack, M. (2005). Utilisation des données ETM+ de Landsat et d'un SIG pour l'évaluation du potentiel en eau souterraine dans le milieu fissuré précambrien de la région de Korhogo (Nord de la Côte d'Ivoire) : Approche par analyse multicritère et test de validation. Télédétection, 5(4), 339-357.

14. Kobenan, C.K., Tia, E.V., Ochou, G.E.C., Kouakou, M., Bini, K.K.N., Dagnogo, M., Dick, A.E., Ochou, O.G. (2018). Comparaison du potentiel insecticide des huiles essentielles de Ocimum gratissimum L. et de Ocimum canum Sims sur Pectinophora gossypiella Saunders (Lepidoptera : Gelechiidae), insecte ravageur du cotonnier en Côte d'Ivoire. European Scientific Journal, Vol.14, No.21, 286-301. http://dx.doi.org/10.19044/esj.2018.v14n21p286 
15. Koffie-Bikpo, C.Y. \& Yéo L. (2016). Maraichage urbain et sécurité sanitaire des aliments à Korhogo. Regards Suds, 176-190. https://regardsuds.org/1945-2

16. Koné, K., Tuo, Y., Yapo, M.L., \& Koua, K.H. (2018). Entomofaune de la courgette (Cucurbita pepo L) en saison pluvieuse, à Korhogo, dans le nord de la Côte d'Ivoire. Int. J. Biol. Chem. Sci., 12(3), 12861297. DOI: https://dx.doi.org/10.4314/ijbcs.v12i3.17

17. Koné, K., Tuo, Y., Yapo, M.L., Soro, F., Traoré, D., \& Koua, K.H., (2019a). Main insect pests of zucchini (Cucurbita pepo L), in the dry season and impact on production in Northern Côte d'Ivoire. Journal of Entomology and Zoology Studies, 7(1), 523-527. http://www.entomoljournal.com/archives/?year=2019\&vol=7\&issue= 1\&ArticleId $=4711$

18. Koné, K., Tuo, Y., Coulibaly, T., \& Koua, K.H. (2019b). Entomofauna and phytosanitary practices in cabbages production (Brassica oleracea L. 1753) in the township of Korhogo of Northern Côte d'Ivoire. IOSR Journal of Agriculture and Veterinary Science, 12 (2), 09-13. DOI: 10.9790/2380-1202010913

19. Kouakou, E., Koné, B., N'Go, A., Cissé, G., Ifejika Speranza, C., Savané, I. (2014). Ground water sensitivity to climate variability in the white Bandama basin, Ivory Coast. SpringerPlus, 3, 226. DOI: 10.1186/2193-1801-3-226

20. Rajendran, T.P., \& Singh D. (2016). Insects and pests. Ecofriendly Pest Management for Food Security, 24p. https://doi.org/10.1016/B978-0-12-803265-7.00001-4

21. Savadogo, S., Sambare, S., Sereme, A., \& Thiombiano, A. (2016). Méthodes traditionnelles de lutte contre les insectes et les tiques chez les Mossé au Burkina Faso. Journal of Applied Biosciences, 105(1), 10120 -10133. http://dx.doi.org/10.4314/jab.v105i1.9

22. Smyth, R.R., \& Hoffman, M.P. (2010). Seasonal incidence of two cooccurring adult parasitoids of Acalymma vittatum in New York State: Centistes (Syrrhizus) diabroticae and Celatoria setosa. BioControl, 55(2), 219-228. https://doi.org/10.1007/s10526-009-9232-y

23. Stevenson, P.C., Dayarathna, T., Belmain, S.R., \& Veitch, N.C. (2009). Bisdesmosidic saponins from Securidaca longepedunculata (Polygalaceae) with deterrent and toxic properties to Coleapteran storage pests. Journal of Agricultural Food Chemistry, 57(19), 88608867. https://doi.org/10.1021/jf901599j

24. Tonzibo, Z.F., Chalchat, J.C., \& N'Guessan, Y.T. (2008). Chemical Composition of Essential Oils of Ocimum canum Sims from Côte d'Ivoire. Journal of Essential Oil Bearing Plants, 11, (5), 530-535. https://doi.org/10.1080/0972060X.2008.10643662 
25. Tuo, Y., Dago, N.D., Yapo, M.L., \& Koua, K.H. (2017). Screening of phytosanitary practices in vegetable Growth Activities Northern of Côte d'Ivoire. Int J Recent Sci Res., 8(6), 17396-17402. https://doi.org/10.1080/0972060X.2008.10643662

26. Tuo, Y., Koné, K., Yapo, L.M., \& Koua, K.H., (2018). Abundance and Incidence of Zucchini (Cucurbita pepo L) Flies in the Korhogo Department of Northern Côte d'Ivoire and Pest Control Methods Used by Farmers. Journal of Experimental Agriculture International, 21(2), 1-7. DOI: $10.9734 / \mathrm{JEAI} / 2018 / 38820$.

27. Zhou, L., Huang, J., \& Xu, H., 2011. Monitoring resistance of field populations of diamondback moth Plutella xylostella L. (Lepidoptera: Yponomeutidae) to five insecticides in South China: A ten-year case study. Crop Prot., 30(3), 272-278. https://doi.org/10.1016/j.cropro.2010.10.006 